\title{
Introduction of Fractional Molar Flux Curves in the Compositional Equations and their Use in Multi-Component Simulations with Coarse Grid in Heterogeneous Media
}

\author{
BARROUX, Claire, Camille \\ RICOIS, Olivier,
}

\author{
Institut Français du Pétrole \\ Beicip-Franlab
}

Paper presented at the 5th European Conference on the Mathematics of Oil Recovery, Leoben, Austria, 3-6 Sept. 1996

\section{ABSTRACT}

Numerical compositional simulation of fully or partially miscible displacements at reservoir scale is known to be adversely affected by severe numerical dispersion arising from the necessity to use coarse grids. Furthermore, fine geological model heterogeneities need to be scaled-up.

The component mass conservation equation has been modified by the introduction of functions for component fractional molar flux. These functions are, as relative permeability functions, characterized by end points, and normalized shapes.

Illustrations of interest in the proposed formalism are mainly given for fully miscible cases. Practical aspects are largely developed.

\section{INTRODUCTION}

Conventional reservoir simulations in the petroleum industry are run with 'coarse' grid blocks to limit the number of cells and CPU time. Upscaling techniques are available to compute the large-scale block properties (porosity, absolute permeability) from the description of the fine-scale geological heterogeneities, and the well productivity indexes. In many cases, it is then possible to simulate the monophasic single-component behaviour of a heterogeneous reservoir with a coarse grid.

For multi-phase and/or multi-component simulations with the most frequently used numerical scheme, - single point upstream weighting for the flow terms -, it is generally necessary to correct the numerical dispersion arising from the large size of the blocks (of the order of hectometers areally, and of one meter to several decameters vertically) : the sharper the saturation/composition front, the bigger the necessity of correcting the convective flux.

In black-oil cases, various techniques have been developed to estimate the pseudo relative permeabilities (kr) to be used at a large scale. When the pseudo $\mathrm{kr}$ are derived from the results of a fine grid model ${ }^{10,18}$, they can simultaneously correct the numerical dispersion and account for the combined effects of fine heterogeneities and the mobility ratio.

When a multicomponent finite difference compositional model is used to simulate miscible displacements at a large scale, individual component material balance equations need to be scaled-up also.

Camy and Emanuel ${ }^{4}$ have proposed techniques for deriving pseudo functions for reducing numerical dispersion and mixing errors : component transport coefficients $\alpha_{k}$ have been introduced into the compositional equations, and equilibrium constants are modified by $\mathrm{K}$-value correction factors $\beta_{\mathrm{k}}$. Fayers et $\mathrm{al}^{8}$ have extended the technique to simulations in heterogeneous media using an equation of state, and proposed a dual zone mixing model of non-uniform phase behaviour involving two flash calculations per grid block. A further improvment, a single flash correction procedure for a comparable accuracy, has been proposed by Barker and Fayers ${ }^{2}$.

The following formulation, written in the case of full miscibility to simplify the presentation, is proposed in the paper :

$\mathbf{J}_{\mathrm{k}}=\overline{\bar{\Gamma}}_{\mathrm{k}} \rho \mathbf{u}$

$\overline{\bar{\Gamma}}_{\mathrm{k}}$ is the tensor of the ' $\mathrm{k}$-component fractional flux functions' ('k-component relative permeability' for Camy). Per direction, $\Gamma_{k}$ and $\alpha_{k}$ are related by :

$\Gamma_{\mathrm{k}}=\alpha_{\mathrm{k}} Z_{\mathrm{k}}$

Symbols are defined in the Nomenclature.

Although initially introduced in the equations at coarse scale, one can note that $\alpha$ correction functions (then $\Gamma$ fractional flux functions) may be used also at a fine scale for particular physical problems. Such a case has been described by Le Thiez ${ }^{12}$. 
Among the modified fractional flux functions already proposed in the literature, one of the most well known is that of $\mathrm{Koval}^{9}$ for unstable two-component miscible displacements, which has been validated at the plug scale. Recently, Lenormand ${ }^{13}$ has proposed, at a large scale, an homogenized transport equation where the fractional flux function is the same as Koval's function.

The proposed formalism can be considered a general way to input various fractional flux functions among which Koval's function is a particular case.

At a large scale, homogenized fractional flux functions are not necessarily the relevant 'pseudo' fractional flux functions to be input into a coarse grid model, since they do not take into account the numerical dispersion. We show how an analytic solution of the convectiondispersion equation can be used to generate fractional flux functions which correct the numerical dispersion. The method is not general, but allows us to get a physical understanding of the $\Gamma$ function behaviour.

As shown hereafter, the $\Gamma$ component fractional flux functions offer some practical advantages, of the same type as those of relative permeability curves, which can be useful to decrease the volume of input data : end points can be defined to normalize the curves ; in fully miscible cases, a same normalized curve can be used for different components. Moreover, curve shapes are smoother than those of $\alpha$-factors since division by 0 (appearence or disappearence of a component) is avoided.

The synthetic cases which are discussed hereafter are :

- a two-component incompressible miscible case (called 'tracer' case )

- two cases of gas recovery by gas injection,

- a case of oil recovery by lean gas injection.

in an either homogeneous monodimensional or heterogeneous areal porous medium.

\section{WORK FRAME}

The numerical simulations are run with a compositional simulation model (ATHOS developed by BEICIP-FRANLAB), using an IMPES scheme and a single point upstream weighting. No water is present.

In each case, the results of a simulation at the fine scale are considered to be the reference data. At the fine scale, the dispersion is purely numerical.

The full fine grid simulations are also regarded as numerical experiments to generate reference $\Gamma$ and $\alpha$ pseudo functions. The method is definitely not practical in reservoir engineering. The interest is to get a better understanding of compositional pseudo function physical contents, useful for handling compositional pseudo functions.

\section{FULLY MISCIBLE FINE 1-D SIMULATIONS}

Generation of compositional pseudo functions from fine grid simulations

From equation (2), the $\Gamma$ functions can be considered an intermediate step in the generation of alpha-factors.
The same generation techniques ${ }^{2,19}$ can be used for both types of tensors.

The ordinate of the $\Gamma$ functions for the coarse block ' $c x$ ' can be computed with :

$$
\left(\Gamma_{\mathrm{k}}^{\mathrm{c}}\right)_{\mathrm{cx}}=\left(\mathrm{z}_{\mathrm{k}}\right)_{\mathrm{fx}}
$$

where ' $f x$ ' is the index of the fine cell, upstream from the outflow boundary of the coarse block ' $c x$ ', and ' $z_{k}$ ' is the $\mathrm{k}$-component molar concentration in the fine cell ' $\mathrm{f} x$ '.

The corresponding $\alpha$-factor functions are :

$\left(\alpha_{\mathrm{k}}^{\mathrm{c}}\right)_{\mathrm{cx}}=\frac{\left(\mathrm{z}_{\mathrm{k}}\right)_{\mathrm{fx}}}{\left(\mathrm{Z}_{\mathrm{k}}\right)_{\mathrm{cx}}}$

which are those introduced by Camy ad Emanuel ${ }^{4} . Z_{k}$ is the k-component molar concentration in the coarse block 'cx', calculated from the concentrations of the subjacent fine blocks.

The pseudo functions derived in such a way have been labeled ' $C$ ' ('Camy' type). It can be noted that they satisfy the relationship :

$\sum_{k}\left(\Gamma_{k}^{c}\right)_{c x}=1$

which can be used for reducing of one the number of $\Gamma$ function sets.

Alternatively, the ordinate of the $\Gamma$ functions can be computed with :

$\left(\Gamma_{\mathrm{k}}^{\mathrm{B}}\right)_{\mathrm{cx}}=\frac{\left(\mathrm{z}_{\mathrm{k}} \rho\right)_{\mathrm{fx}}}{(\rho)_{\mathrm{cx}}}$

where $\rho$ and $\rho$ are fluid molar density, respectively at fine and coarse scale.

They include then the modification proposed by Barker and Fayers for the $\alpha$-factors :

$\left(\alpha_{k}^{B}\right)_{c x}=\frac{\left(z_{k} \rho\right)_{\mathrm{kx}}}{\left(Z_{k} \rho\right)_{\alpha x}}$

These pseudo functions have been labeled ' $B$ ' ('Barker' type).

\section{End-point definition}

As a fluid 1 is injected to displace a fluid 2, the minimum and maximum values of the range of concentration variations are obtained from the initial compositions of the fluids 1 and 2 . As an example, if a gas, the molar composition of which is $90 \%$ methane $(\mathrm{Cl})$ and $10 \%$ butane $(\mathrm{C} 4)$, is injected to recover a heavier gas (75\% Cl- 16\% C4 - 9\% C10), the concentration endpoints are :

- $Z_{1 \min }=0.75 ; Z_{1 \max }=0.90$

- $Z_{4 \min }=0.10 ; Z_{4 \max }=0.16$

- $Z_{10 \min }=0.00 ; Z_{10 \max }=0.09$

Normalized concentrations and fractional flux can then be defined :

$Z_{k}^{\text {norm }}=\frac{Z_{k}-Z_{k \min }}{Z_{k \max }-Z_{k \min }}$ 


$$
\Gamma_{k}^{\text {norm }}=\frac{\Gamma_{k}-Z_{k \min }}{Z_{k \max }-Z_{k \min }}
$$

End points, depending on the in-place fluid composition, are data of thermodynamic type.

\section{Simulated Cases}

The fine and coarse grids (aggregation rate equal to 9) used for the numerical simulations are presented table 1 and figure 1 . The cases differ only in the composition of the injected and the in-place fluids. The injector works at a constant rate and the producer at a constant limiting bottom-hole pressure, equal to the initial reservoir pressure.

The calculation time step is kept far below the stability limit.

\section{'tracer' case}

At the inlet face of an incompressible homogeneous medium and at $t=0$, a single component ' $\mathrm{t}$ ' fluid is injected at a constant filtration velocity to displace a single component ' $s$ ' fluid initially in place. Both components have the same physical properties, are incompressible and are fully miscible. Given these particular features, no equation of state has been used.

\section{' $L G$ ' case}

Pure methane ('L'ean 'G'as) is injected to displace a gas, the composition of which is $75 \mathrm{~mol} \%$ methane, $16 \mathrm{~mol} \%$ $\mathrm{n}$-butane and $9 \mathrm{~mol} . \%$ decane. Thermodynamic conditions are given in table 2. Thermodynamics are based on Peng Robinson's equation of state.

\section{' $R G$ ' case}

The difference between the 'RG' ('R'ich 'G'as) case and the former case is the composition of the injected gas, 90 mol.\% methane and $10 \mathrm{~mol} . \% \mathrm{n}$-butane.

\section{Results}

The functional dependence of the normalized $\mathrm{C}_{\mathrm{k}}$ norm ('Camy' type) fractional flux with $Z_{k}$ norm , obtained from the ' $L G$ ' and 'RG' fine simulations, is presented respectively in figures 2 and 3, for three macro-cells, together with those from the 'tracer' case. It can be noted that :

- the components, the concentration of which increases with time, have the same normalized $\Gamma^{\mathrm{c}}$ curve, which is the $\Gamma_{t}$ curve of the 'tracer' case.

- the components, the concentration of which decreases with time, have also a same normalized $\Gamma^{c}$ curve (the $\Gamma_{\mathrm{s}}$ curve of the 'tracer' case). At the minimum concentration, the slope of the curve is greater than one. If, during the simulation, one of these components disappears, the corresponding $\alpha$-factors may reach large values.

- the pseudos are position-dependent.

As the fractional flux curves depend on the concentration path (increasing/decreasing), hysteresis effects can be expected with variation in the injected fluid composition.
A set (macro-cell 4) of $\alpha_{k}^{c}$ curves (no normalization of ordinate) is shown in figure 4, for 'tracer', 'LG' and 'RG' cases. As expected for the components, the concentration of which decreases, $\alpha$-factors exhibit a singular behaviour at component disappearance.

$$
\text { c }
$$

The $\alpha$-factors are strongly case-sensitive, as the same normalized $\Gamma^{c}$ functions can be directly transferred from one case to the next. Such a property is very useful when running sensitivity studies, for instance to the composition of the injected gas ; it can also be used to recalculate the $\alpha$-factors, in the case where this formalism is already implemented in the available simulator, thus avoiding the rerun of fine grid simulations.

Figure 5 shows the normalized fractional flux functions calculated using equation (6), for ' $L G$ ' and 'RG' cases ; the 'tracer' curves are also plotted to help the comparison. One can see that the normalized $\Gamma^{B}$ functions are casesensitive and that, in the ' $R G$ ' case, each component normalized curve is differenciated. Although more accurate to upscale the convective term, the $\Gamma^{\mathrm{B}}$ functions appear more difficult to handle, and are to be kept only when a significant improvement is expected from their use.

\section{ANALYTICAL 1-D SIMULATION}

Aronofsky and Heller ${ }^{1}$ derived an analytical solution to the monodimensional convection-diffusion equation :

$\partial_{t}\left(\mathrm{z}_{1}\right)+\mathrm{v} \partial_{x}\left(\mathrm{z}_{1}-\mathrm{d} \partial_{x}\left(\mathrm{z}_{t}\right)\right)=0$

which is applicable for a problem similar to the 'tracer' case presented hereabove ; the only difference is that the homogeneous monodimensional porous medium is semiinfinite and physically dispersive :

$$
\begin{aligned}
\mathrm{z}_{\mathrm{t}}(x, t)=\frac{1}{2}\{ & {\left[1-\operatorname{Erf}\left(\frac{x-\mathrm{v} t}{2 \sqrt{\mathrm{d} \mathrm{v} t}}\right)\right] } \\
& \left.+\operatorname{Exp}\left(\frac{x}{\mathrm{~d}}\right)\left[1-\operatorname{Erf}\left(\frac{x+\mathrm{v} t}{2 \sqrt{\mathrm{d} \mathrm{v} t}}\right)\right]\right\} \ldots . . .(I I)
\end{aligned}
$$

To make the analogy between the numerical and analytical simulations, the physical dispersivity ' $d$ ' has been taken as the numerical dispersivity issued from Lantz's work ${ }^{11}$ :

$\mathrm{d}=\frac{\delta x-\mathrm{v} \delta t}{2}$

where $\delta x$ is the dimension of the fine grid, and $\delta t$, the calculation time step.

At a time $t$, the average concentration between $x_{\mathrm{m}}-\Delta X / 2$ and $x_{\mathrm{m}}+\Delta X / 2$ ( $\Delta X$ being the dimension of the coarse grid) is given by :

$Z_{\mathrm{t}}\left(x_{\mathrm{m}}, t\right)=\frac{1}{\Delta X} \int_{x_{\mathrm{m}}-\Delta X Z 2}^{x_{\mathrm{m}}+\Delta X Z 2} \mathrm{z}_{\mathrm{l}}(x, t) \mathrm{d} x$

This represents the average concentration of a block, the center of which is located at a distance $x_{\mathrm{m}}$ from the injector. 
From equation (10), the ' $t$ ' component fractional flux issuing the block centered on $x_{\mathrm{m}}$ is :

$\Gamma_{\mathrm{t}}\left(x_{\mathrm{m}}+\Delta X / 2, t\right)=\mathrm{z}_{\mathrm{l}}\left(x_{\mathrm{m}}+\Delta X / 2, t\right)-\mathrm{d} \frac{\partial}{\partial x} \mathrm{z}_{\mathrm{t}}\left(x_{\mathrm{m}}+\Delta X / 2, t\right) \ldots(14)$

The curve $\Gamma_{\mathrm{t}}\left(x_{\mathrm{m}}+\Delta X / 2, t\right)$ versus $Z_{\mathrm{t}}\left(x_{\mathrm{m}}, t\right)$ is the analytically derived $\Gamma_{t}$ function, easily computable using a spreadsheet. In figure 6 , for three macro-cell locations, the analytically derived $\Gamma$ functions are compared to those obtained from the fine grid simulation.

One can observe that the pseudo fractional flux curves are reproduced quite well.

Along with the dependence on the position of the cell center, we used the analytical simulation to analyze the

dependence of the fractional flow curves on the ratio $\frac{\Delta X}{2 \mathrm{~d}}$ (figure 7), which, in the analogy with a numerical experiment, is equivalent to the aggregation ratio $\frac{\Delta X}{\delta x}$ for small calculation time steps. Attempting to fit the curve shapes with a power law:

$\Gamma_{1}\left(Z_{t}\right)=Z_{t}^{\kappa}$

we found an empirical explicit form of the exponent :

$\kappa=\sqrt{1+\sqrt{2} \frac{\Delta X}{2 \mathrm{~d}} \frac{\Delta X}{x_{\mathrm{m}}}}$

which allows for reproducing the dependence of the fractional flux function $\Gamma_{\mathrm{t}}\left(\mathrm{Z}_{\mathrm{t}}\right)$ on $\Delta X, \mathrm{~d}$, and $x_{\mathrm{m}}$ approximately, as $\mathrm{d}$ is lower than $\Delta X / 2$. This helps us for understanding what parameters influence the curve shape of the injected component fractional flux.

A first direct conclusion is that it is advisable to use regular grids to limit the number of required pseudos. Besides, it appears that the physical rock-type parameter, which controls intrinsically the curve shapes, should be a physical dispersivity coherent with the scale adopted for the homogenization of the absolute permeabilities.

The main problem is that, as for dynamic pseudo relative permeabilities ${ }^{3}$, the compositional pseudo fractional flux are found position-dependent. In a one-dimensional simulation, the parameter $x_{\mathrm{m}}$, the distance of the cell from the injector, is well defined and position-dependent pseudo functions could be used ${ }^{17}$, with one set of pseudo functions per grid block. However, the use of positiondependent pseudos is not convenient, especially in multiwell multi-dimensional applications. The only solution which has been found to avoid the use of multiple (position-dependent) sets of $\Gamma$ functions is empirical. In what follows, a same set of pseudo fractional flux was used for all coarse grid blocks, except in one coarse simulation run in order to test the implementation of the fractional flux formalism in the simulator. The so-called 'mean' set of fractional molar flux corresponds to the fractional flux curves of a coarse block located at middistance from the wells. It is shown in figure 9 .

\section{FULLY MISCIBLE COARSE 1-D SIMULATIONS}

\section{'tracer' case}

The 'reference' concentration profile (from the fine grid simulation) at the producer is shown in figure 8 together with those from three coarse simulations :

- without any pseudo fractional flux curve, (profile (1)),

- with the 'mean' set of $\Gamma$ functions (figure 9), (profile (2)),

- with an artificial data modification, (profile (3)), which is discussed hereafter.

In the first case, the front mean velocity (inflexion point) is reproduced, but not the breakthrough time, because of the numerical dispersion which enlarges the front width. As the 'mean' functions are used, the shape of the concentration front is reproduced well. But the front mean velocity is underestimated despite the fact that the interwell distance is strictly conserved from the fine to the coarse simulation. The same observation has been made in the 2-D simulation described hereafter. This problem is not particular to the fractional flow formalism, as it was encountered in an earlier work, not mentionned here, concerning the dynamic relative permeability upscaling. It is due to the use of a 'mean' set of pseudos. The time discrepency $\tau$ between the coarse and fine simulations corresponds to a distance $\left(\frac{u}{\phi} \tau\right)$, found nearly equal to the size of the coarse grid block $\Delta X$. In the 1-D simulation, we found that the introduction of specific fractional flux functions for perforated cells allows for a quasi-exact reproduction of the production concentration profile : in these pseudos, the unswept 'dead' volumes of the perforated coarse cells are considered through a 'trapped' concentration (figure 10). Nevertheless, such a procedure limited to the perforated cells was found to be unsufficient in the 2-D simulations, a global data modification having to be envisaged to get the proper time scale. In particular cases (uniform porosity, regular isotropic grid) presented here, there are various artificial ways to correct the discrepency, among which the following allow for the quantitative reproduction of the production concentration profiles :

- keep the 'mean' set of $\Gamma$ functions unchanged and use at the coarse scale, in a very arbitrary way, a modified value for the uniform porosity. The results (profile (3) of figure (8)) are superimposed on the reference data. The ratio between the modified and initial porosity values is $7 / 8$, or $(\mathrm{N}-1) / \mathrm{N}(\mathrm{N}$ is the number of coarse blocks).

- renormalize the 'mean' set of $\Gamma$ functions with a 'trapped' concentration (figure 11) equal to $1 / 8$, the disadvantage being that the concentration maps are biased ; the production concentration profile remains practically the same as in the previous method.

Although the research of a general, physically acceptable, solution to this problem is beyond of the scope of this paper, it is believed that an appropriate way to use 'mean' (non position-dependent) pseudos could be the introduction, in the convective term of material balance 
equations, of a kind of 'conformance' factor (griddependent, directional, but not composition dependent). Here, only artificial biases have been used.

\section{' $L G$ ' and ' $R G$ ' cases}

The results are presented in figures 12 and 13. As for the 'tracer' case, the results of three coarse simulations are shown together with the 'reference' concentration profile (from the fine grid simulation) :

- without any pseudo fractional flux curve, (profile (1)),

- with a 'mean' normalized set of $\Gamma$ functions identical to the 'mean' set of $\Gamma$ functions (figure 9) for the tracer case), and case-dependent end-points (profile (2)),

- with the above definition of $\Gamma$ functions and the same modification of the porosity as in the tracer case (profile (3)).

It can be seen that the results are in concordance with the former discussion and illustrate, through the use of normalized.pseudos, the interest of the proposed formalism.

\section{FULLY MISCIBLE 2-D SIMULATIONS}

The reservoir is a horizontal layer, of uniform porosity with one injector and two producers. The spatially correlated random permeability distribution was generated using the Turning Bands Method ${ }^{15}$ with a correlation length in the range of interwell distance and a variance $\left(\sigma^{2} \ln (k)\right)$ of 1 . The permeability map, together with well locations, is shown in figure 15 . The mean permeability value is the permeability used in the $1-D$ model. Both fine $(108 \times 108)$ and coarse $(12 \times 12)$ grids are regular and isotropic, with block dimensions respectively of 5 meters and 45 meters. The wells work in the same way as in the 1-D simulations. Two fine grid simulations are presented below, corresponding to the 'tracer' and 'LG' cases discussed above.

For multi-dimensional grids, the numerical dispersion was quantified by Fanchi ${ }^{\top}$. In areal geometry, high fluid velocities are only met in well vicinities. Using an isotropic grid, for most of the coarse grid cells, the product $(\mathrm{v} \delta t)$ is small compared to $\Delta X$. As a result, the simulations are not strongly sensitive to the calculation time step and the numerical dispersivity is approximately the numerical dispersivity of a 1-D simulation run with the same grid size and a small calculation time step.

\section{Permeability and productivity index upscaling}

Among the methods for permeability up-scaling ${ }^{16}$, Le Loc'h and Guérillot's estimator ${ }^{14}$ :

$\mathrm{K}_{\text {eq }}=\sqrt{\mathrm{K}^{+} \mathrm{K}^{-}}$

where $\mathrm{K}^{+}$and $\mathrm{K}^{-}$are the absolute permeability minima and maxima defined by Cardwell and Parsons ${ }^{5}$, is suitable for 2D isotropic conditions. This was used to evaluate the coarse block connection permeabilities.

The productivity indexes on the coarse grid are obtained directly from the 'tracer' case permanent flow using Ding's method'.

\section{Simulated Cases}

'tracer' case

The permanent producer rates obtained from the coarse simulation are very close to the 'reference' (fine grid simulation) values :

$$
\begin{aligned}
\text { - } Q_{\mathrm{P} 1}^{\text {fine }} & =8.675 \mathrm{~m} 3 / \mathrm{d}, Q_{\mathrm{Pl}}^{\text {coarse }}=8.54 \mathrm{~m} 3 / \mathrm{d} \text {, } \\
\text { fine } & =31.325 \mathrm{~m} 3 / \mathrm{d}, Q_{\mathrm{P} 2}^{\text {coarse }}=31.46 \mathrm{~m} 3 / \mathrm{d} \text {, }
\end{aligned}
$$

This confirms the choices for permeability and IP upscaling.

The fine grid results were used to generate reference $\Gamma$ pseudo functions per cell and per direction. The $2 \times 12 \times$ 11 sets of reference curves were reduced to 90 tables by keeping the reference curves (then the position dependence) in the zones of high velocity and by grouping the curves of low velocity zones in an empirical manner. These pseudos were used in a coarse simulation run to validate the fractional flux formalism. The reference data were reproduced (figure 14).

In figure 16 , the three coarse simulations (1), (2) and (3) are shown. The fractional flux curves are the same as those used in the corresponding 1-D simulations. The results of case (3) (same artificial porosity reduction as before) are in good agreement with the reference data.

Indeed, the scale used for permeability homogeneisation being far below the correlation length, we could have expected that the effects of the physical dispersion resulting from permeability upscaling be of second order compared to the convective effects ${ }^{3}$, and that the 1-D analytical model (based only on the numerical dispersion) be relevant for providing an acceptable 'a priori' estimation of the 'mean' set of $\Gamma$ functions.

\section{' $L G$ ' case}

The same types of coarse simulations as before (still the same 'mean' set of normalized $\Gamma$ functions) were run with the same types of results. In figure 17 , the rate history of both producers and their production concentration profile are presented respectively. Even if the agreement of profile (3) with reference data is not as good as for the tracer case, this coarse simulation is much more predictive than the coarse simulation without any fractional flux function (profile (1)).

\section{PARTIALLY MISCIBLE 1-D SIMULATION}

As two hydrocarbon phases are present, the $\Gamma$ functions can be defined per phase as :

$$
\left(\Gamma_{\mathrm{k}}^{\mathrm{B}}\right)_{\mathrm{cx}}^{\mathrm{O}}=\frac{\left(\mathrm{x}_{\mathrm{k}} \rho_{\mathrm{e}}\right)_{\mathrm{Fx}}}{\left(\rho_{\mathrm{o}}\right)_{\mathrm{cx}}} \text { and }\left(\Gamma_{\mathrm{k}}^{\mathrm{B}}\right)_{\mathrm{cx}}^{\mathrm{g}}=\frac{\left(\mathrm{y}_{\mathrm{k}} \rho_{\mathrm{g}}\right)_{\mathrm{Ex}}}{\left(\rho_{\mathrm{g}}\right)_{\mathrm{cx}}}
$$

or :

$\left(\Gamma_{k}^{c}\right)_{a x}^{o}=\left(x_{k}\right)_{f x}$ and $\left(\Gamma_{k}^{c}\right)_{c x}^{g}=\left(y_{k}\right)_{f x}$

where ' $x_{k}$ ' and ' $y_{k}$ ' are the k-component concentrations, in oil and gas phase respectively, in the fine cell ' $\mathrm{f} x$ '.

The various choices for defining the functional dependency of the $\Gamma$ functions are then the same as those 
already reported by Barker et al. $^{2}$ concerning the $\alpha$ factors.

Alternatively, one can define $\Gamma$ functions common to both phases:

$$
\left(\Gamma_{k}^{B}\right)_{c x}=\frac{f_{0}\left(x_{k} \rho_{0}\right)_{\mathrm{fx}}+f_{\mathrm{g}}\left(y_{k} \rho_{g}\right)_{\mathrm{fx}}}{f_{\mathrm{o}}\left(\rho_{\mathrm{o}}\right)_{\mathrm{cx}}+\mathrm{f}_{\mathrm{g}}\left(\rho_{\mathrm{g}}\right)_{\mathrm{cx}}}
$$

where $f_{o}$ and $f_{g}$ are oil and gas fractional flux respectively. The corresponding 'Camy' type function :

$\left(\Gamma_{\mathrm{k}}^{\mathrm{c}}\right)_{\mathrm{cx}}=\frac{\mathrm{f}_{\mathrm{o}}\left(\mathrm{x}_{\mathrm{k}} \rho_{\mathrm{o}}\right)_{\mathrm{fx}}+\mathrm{f}_{\mathrm{g}}\left(\mathrm{y}_{\mathrm{k}} \rho_{\mathrm{g}}\right)_{\mathrm{fx}}}{\mathrm{f}_{\mathrm{o}}\left(\rho_{\mathrm{o}}\right)_{\mathrm{fx}}+\mathrm{f}_{\mathrm{g}}\left(\rho_{\mathrm{g}}\right)_{\mathrm{fx}}}$

represents the overall outflowing molar concentration of the k component.

Using the 1-D fine grid model already presented, we simulated a methane injection into a three-component oil in conditions of partial miscibility (case ' $O$ ' of table 2), and generated the various types of $\Gamma$ functions.

In figure 19 , the methane $\Gamma^{c}$ functions (oil and gas) obtained from equation (19), are plotted versus up-stream coarse block component concentration in the considered phase (obtained from the flash of its overall contents) : $\left(\Gamma_{\mathrm{Cl}}^{\mathrm{B}}\right)^{\mathrm{o}}$ versus $\mathrm{X}_{\mathrm{Cl}}$ and $\left(\Gamma_{\mathrm{Cl}}^{\mathrm{B}}\right)^{\mathrm{g}}$ versus $\mathrm{Y}_{\mathrm{Cl}}$. During the oil swelling phase, the $\mathrm{Cl}$ concentration in oil increases. At the saturation point, a gas saturation appears and the $\mathrm{Cl}$ concentration in oil decreases. The $\left(\Gamma_{\mathrm{Cl}}^{\mathrm{B}}\right)$ function is then multi-valued and not of simple use.

In figure 18, the various $\Gamma^{c}$ functions from equations (19) and (21), are plotted versus the overall concentration of the considered component. Their shapes result from the various thermodynamic steps. Each curve is different from one component to the next. Hence, there is no longer any advantage in choosing $\Gamma^{\mathrm{C}}$ rather than $\Gamma^{\mathrm{B}}$ functions. Nonetheless, curve normalization, helpful when tabulating input data since the variation range is the same for all components, is still possible.

The results of numerical simulations are shown in figure 20. In coarse simulation (1), one set of pseudo kr (obtained for macro-cell 4 from Stone's method ${ }^{18}$ ) is used without any $\Gamma$ function nor porosity modification. In coarse simulation (3), the same pseudo kr were used, the porosity was modified (as for fully miscible cases), and the pseudo fractional flux curves common to oil and gas shown in figure 18 were used. The porosity modification allows for matching the gas breakthrough time. The main effect of $\Gamma$ functions is on the composition of produced fluids. They allow the reproduction of the compositions until gas breakthrough, but, afterwards, the profiles are distorted. The same types of results were obtained using separate curves for oil and gas. To avoid any conflict, as two hydrocarbon phases are flowing, between the pseudo $\mathrm{kr}$ and $\Gamma$ functions (both composition dependent), it could be envisaged, through a test on the number of flowing hydrocarbon phases, to activate the $\Gamma$ functions only when the hydrocarbon outflow is monophasic.

\section{IMPLEMENTATION}

For the functional dependency, we chose to refer each ' $\Gamma_{\mathrm{k}}$ ' value to the upstream coarse block concentration of the same component ' $Z_{k}$ '. Although only tested with an IMPES scheme, such a functional dependence should allow a fully implicit implementation in fully miscible cases. For partially miscible cases, a fully implicit implementation appears difficult, especially if, through a test on the number of flowing phases (offered to the user as an option), the $\Gamma$ functions are only activated when the hydrocarbon outflow is monophasic.

Currently, the $\Gamma$ functions are entered as recurrent data in a tabular form. However, for normalized shapes in fully miscible cases, some simple analytical forms (exponential type, Koval, ..), described by a limited number of parameters, could be a useful alternative.

\section{CONCLUSIONS}

1- The introduction of fractional molar flux functions into the compositional equations is an alternative to the $\alpha$ factor formalism for improving the accuracy of compositional simulations.

2- The proposed formalism is shown to provide a base for understanding the problems of compositional upscaling.

3- It allows for reducing the volume of input data :

- in cases of continuous fully miscible injection, a multi-component system is shown to be reducible to a 2-component system through the normalization of the fractional molar flux functions, when a simplified definition ('Camy') is used.

- in partially miscible cases, one can define $\Gamma$ functions common to oil and gas phases.

4- An analytical method for an 'a priori' estimation of the normalized shapes in fully miscible cases is described and has been used successfully in 2-D areal simulations in a heterogeneous medium.

5- In simple cases, the use of non position-dependent pseudos is shown to be possible, but to require either a code modification or artificial biases.

6- Extensions of this work are needed :

- to optimize the application rules in partially miscible cases and in cases of compositional variations at injection,

- to explore grid orientation effects and threedimensional applications.

\section{NOMENCLATURE}

When two symbols are given on the same line, the first is used for coarse scale and the second for fine scale.

$\begin{array}{ll}\text { bold } & : \text { vector } \\ \mathrm{d} & : \text { dispersivity } \\ \Delta X, \delta x & : \text { grid-block dimension (x direction) } \\ \mathrm{f} & : \text { phase fractional flux } \\ \mathrm{J} & : \text { convective flux } \\ \mathrm{K} & : \text { absolute permeability } \\ t & : \text { time }\end{array}$




$\begin{array}{ll}\mathbf{u} & : \text { Darcy velocity } \\ \mathrm{v} & \text { : phase mean velocity }(\mathrm{u} / \phi, \mathrm{x} \text { direction }) \\ x & \text { : distance from the injection point } \\ \mathrm{X}, \mathrm{x} & : \text { upstream cell oil phase mol. fraction } \\ \mathrm{Y}, \mathrm{y} & : \text { upstream cell gas phase mol. fraction } \\ \mathrm{Z}, \mathrm{Z} & \text { : upstream cell overall mol. fraction } \\ \alpha & : \text { transport coefficient } \\ \Gamma & : \text { fractional molar flux } \\ \phi & : \text { porosity, fraction } \\ \rho, \rho & : \text { molar fluid density }\end{array}$

\section{Subscripts}

$\mathrm{cx}, \mathrm{fx} \quad$ : cell index ( $\mathrm{x}$ direction)

g : gas phase

$\mathrm{k} \quad$ : component index ( general ; ' $\mathrm{t}$ ' and ' $\mathrm{s}$ ', subscripts are used in the 'tracer' case)

$\begin{array}{ll}\text { o } & \text { : oil phase } \\ \text { C } & \text { : 'Camy' type } \\ \text { B } & \text { : 'Barker' type }\end{array}$

\section{ACKNOWLEDGMENTS}

Part of this work was carried out in the context of an 'ARTEP' project. The authors thank Elf Aquitaine Production, Total, and Institut Français du Pétrole for their permission to publish this paper. Special thanks are addressed to $\mathrm{L}$. Weill for assistance in programming.

\section{REFERENCES}

1. Aronofsky, J.S. and Heller, J.P. : «A Diffusion Model to Explain Mixing of Flowing Miscible Fluids in Porous Media », Trans. AIME, 210, 1957, p.345.

2. Barker, J.W. and Fayers, F.J. : "Transport Coefficients for Compositional Simulation With Coarse Grid in Heterogeneous Media ", SPE 22591, 66th SPE Annual Technical Conference \& Exhibition, Dallas, U.S.A., Oct. 1991.

3. Barker, J.W. and Thibeau S. : "A Critical Review of the Use of Pseudo Relative Permeabilities for Upscaling ", SPE 35491, European 3-D Reservoir Modelling Conference, Stavanger, Norway, Apr. 1996.

4. Camy, J.P. and Emanuel, A.S. : « Effect of Grid Size in the Compositional Simulation of CO2 Injection ", SPE 6894, 52nd SPE Annual Technical Conference \& Exhibition, Denver, U.S.A., Oct. 1977.

5. Cardwell, W.T. Jr. and Parsons, R.L. Jr. : «Average Permeabilities of Heterogeneous Oil Sands", Trans. AIMM Pet. Eng., 160, 1945, p.34.
6. Ding, Y.: " Scaling-Up in the Vicinity of Wells in Heterogeneous Field », SPE 29137, 13th SPE Symposium on Reservoir Simulation, San Antonio, U.S.A., Feb. 1995.

7. Fanchi, J.R. : «Multidimensional Numerical Dispersion ", $S P E J$ (Feb. 1983) 143.

8. Fayers, F.J., Barker, J.W. and Newley, T.M.J. : « Effects of Heterogeneities on Phase Behaviour in Enhanced Oil Recovery ", Euro. Conference on the Math. of Oil Recovery, Cambridge, U.K., July 1989.

9. Koval, E.J. : «A Method for Predicting the Performance of Unstable Miscible Displacement in Heterogeneous Media », SPEJ (June 1963) 145 ; Trans. AIME, 228.

10. Kyte, J.R. and Berry, D.W. : « New Pseudo Functions to Control Numerical Dispersion ", SPEJ (Aug. 1975) 269.

11. Lantz, R.B. : "Quantitative Evaluation of Numerical Diffusion (Truncation Error) », SPEJ (Sept. 1971) 315.

12. Le Thiez, P. and Ducreux J. : " A 3-D Numerical Model for Analyzing Hydrocarbon Migration into Soils and Aquifers ", 8th International Conference on Computer. Methods and Advances in Geomechanics, Morgantown, U.S.A., May 1994.

13. Lenormand, R. : «Transport Equations for Fluid Displacements in Stratified Porous Media : the MHD Model , SPE 30797, SPE Annual Technical Conference \& Exhibition, Houston, U.S.A., Oct. 1995.

14. Le Loc'h, G. : «An Efficient Strategy for Combining the Permeabilities. Practical Application on a Simulated Reservoir », 3rd International Geostatistics Congress, Avignon, Kluwer Acad. Pres. 1989.

15. Mantoglou, A. and Wilson, J.L. : "The Turning Bands Method for Simulation of Random Fields using Line Generation by a Spectral Method ", Water Resources Research, 18 (5) , 1982, p.1379.

16. Romeu R.K. : « Ecoulement en milieux hétérogènes : prise de moyenne de perméabilité en régimes transitoire et permanent ", PhD Thesis, Université de Paris VI, June 1994.

17. Soedarmo E., Taggart I.J. and Paterson L. : « LengthDependent Pseudofunction : An Improved Up-scaling Method in Black Oil Simulation », SPE 28754, SPE Asia Pacific Oil \& Gas Conf., Melbourne, Australia., Nov. 1994.

18. Stone H.L. : "Rigorous Black Oil Pseudo Functions 》, SPE 21207, 11th Symposium on Reservoir Simulation, Anaheim, U.S.A., Feb. 1991.

19. Thibeau S., Barker, J.W. and Souillard Ph. : " Dynamical Upscaling Techniques Applied to Compositional Flows ", SPE 29128, 13th Symposium on Reservoir Simulation, San Antonio, U.S.A., Feb. 1995.

\section{TABLES}

\begin{tabular}{|c|c|c|c|c|c|}
\hline \multicolumn{6}{|c|}{ Table 1 - 1-D Simulation Grids } \\
\hline Grid & $\begin{array}{l}\text { Number } \\
\text { of cells }\end{array}$ & $\begin{array}{l}\text { Grid } \\
\text { size } \\
(\mathrm{m})\end{array}$ & $\begin{array}{c}\mathrm{K} \\
(\mathrm{mD}) \\
\end{array}$ & $\begin{array}{c}\phi \\
(\%)\end{array}$ & $\begin{array}{c}\text { Distance } \\
\text { inject.-prod. } \\
\text { (m) }\end{array}$ \\
\hline Fine & 72 & 5 & 239.6 & 20 & 315 \\
\hline Coarse & 8 & 45 & 239.6 & 20 & 315 \\
\hline
\end{tabular}

\begin{tabular}{|c|c|c|c|c|c|c|c|}
\hline \multicolumn{7}{|c|}{ Table 2 - Thermodynamic conditions } \\
\hline Case & $\mathrm{T}$ & $\mathrm{P}$ & \multicolumn{2}{|c|}{ Injected Gas } & \multicolumn{3}{|c|}{$\begin{array}{c}\text { Fluid } \\
\text { initially in place } \\
\text { (mol. \%) }\end{array}$} \\
& $\left({ }^{\circ} \mathrm{C}\right)$ & (bar) & \multicolumn{2}{|c|}{ (mol. \%) } & \multicolumn{3}{|c|}{$\begin{array}{c}\text { (m) } \\
\end{array}$} \\
& & & $\mathrm{Cl}$ & $\mathrm{C} 4$ & $\mathrm{Cl}$ & $\mathrm{C} 4$ & $\mathrm{C} 10$ \\
'LG' & 135 & 300 & 100 & 0 & 75 & 16 & 9 \\
'RG' & 135 & 300 & 90 & 10 & 75 & 16 & 9 \\
'O' & 71.1 & 265 & 100 & 0 & 65 & 16 & 19 \\
\hline
\end{tabular}




\section{FIGURES}

Figure 1 - Monodimensional Fine and Coarse Grid

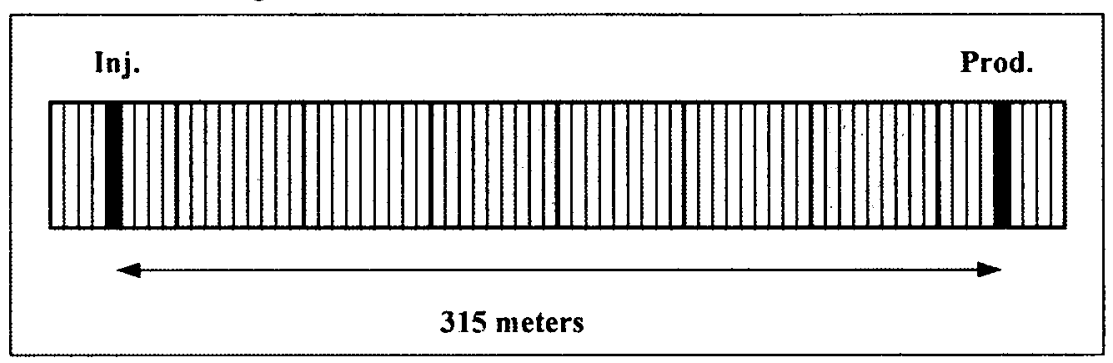

Figure 2 - 1-D 'Tracer' and 'LG' cases : Normalized Fractional Flux Functions (Camy type)
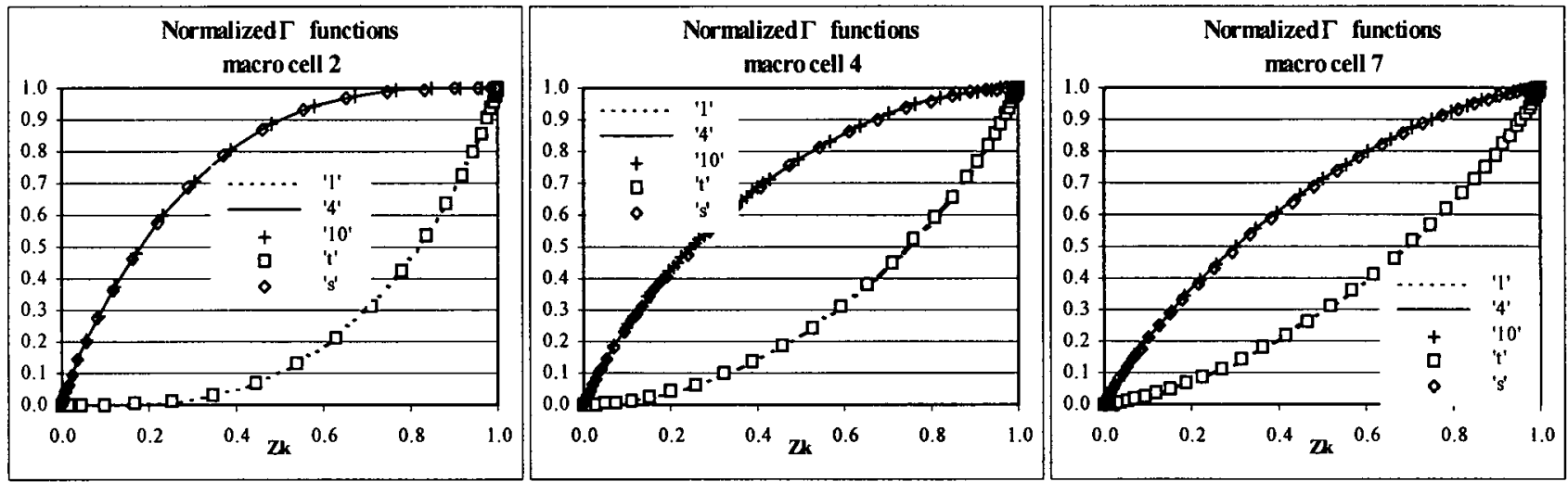

Figure 3 - 1-D 'Tracer' and 'RG' cases : Normalized Fractional Flux Functions (Camy type)
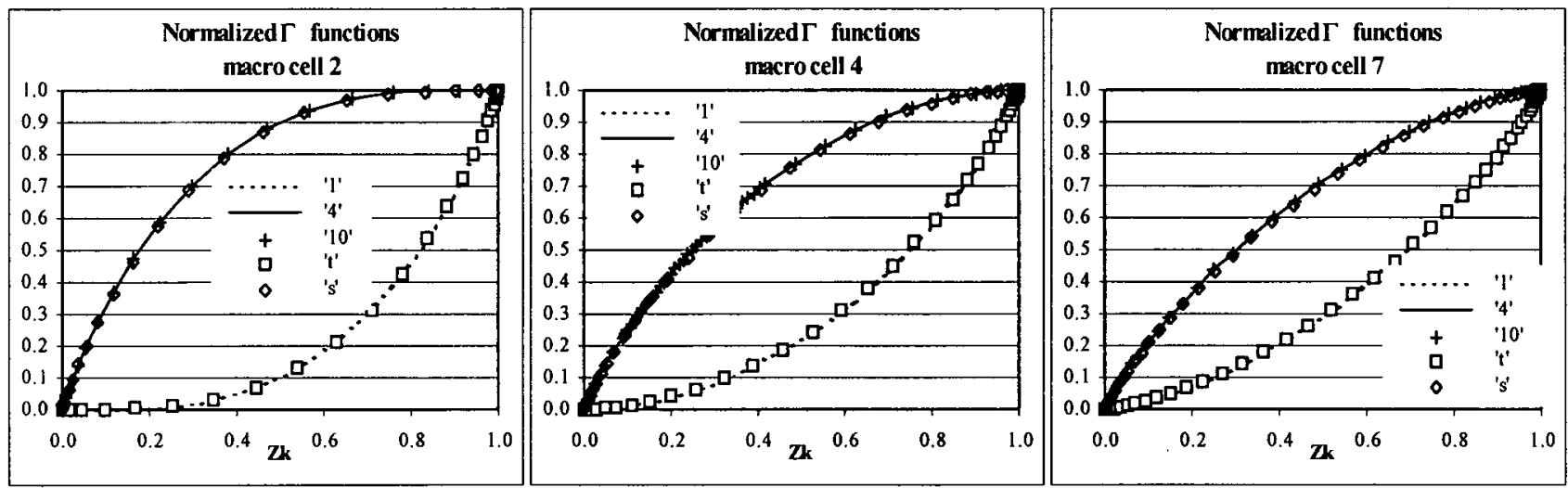

Figure 4 - 1-D 'Tracer', 'RG' and 'LG' cases : Alpha Functions (Camy type)
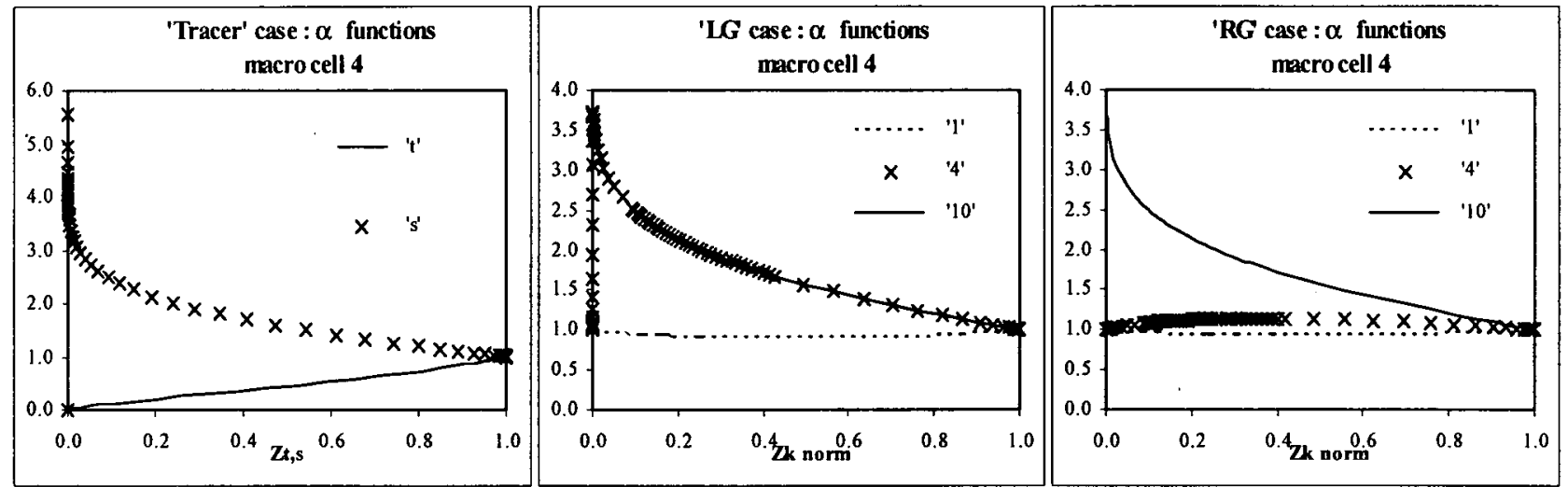
Figure 5 - 1-D ‘LG' and 'RG' cases : Normalized Fractional Flux Functions (Barker type)

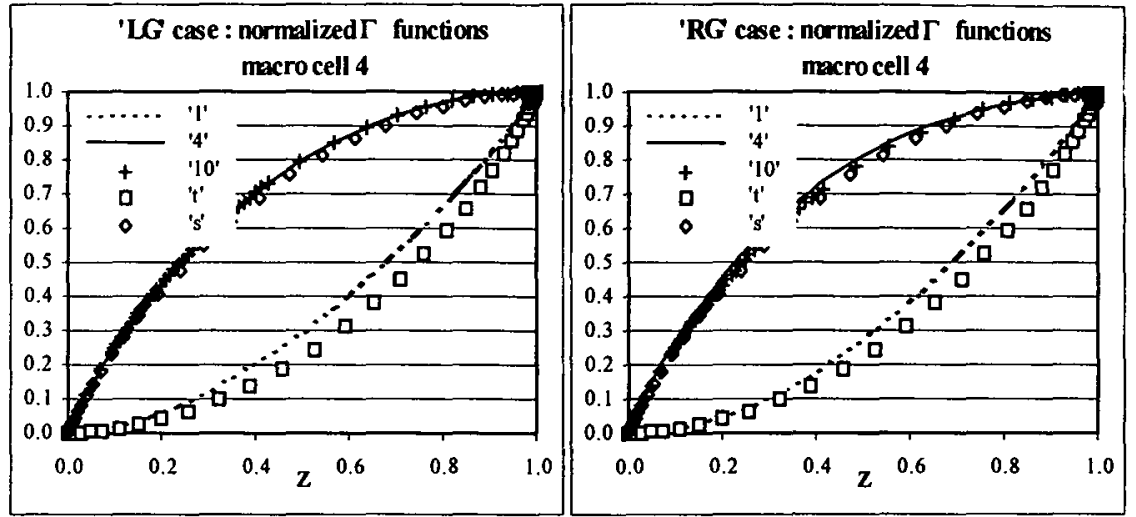

Figure 6 - 1-D 'Tracer' Case : Fractional Flux Functions

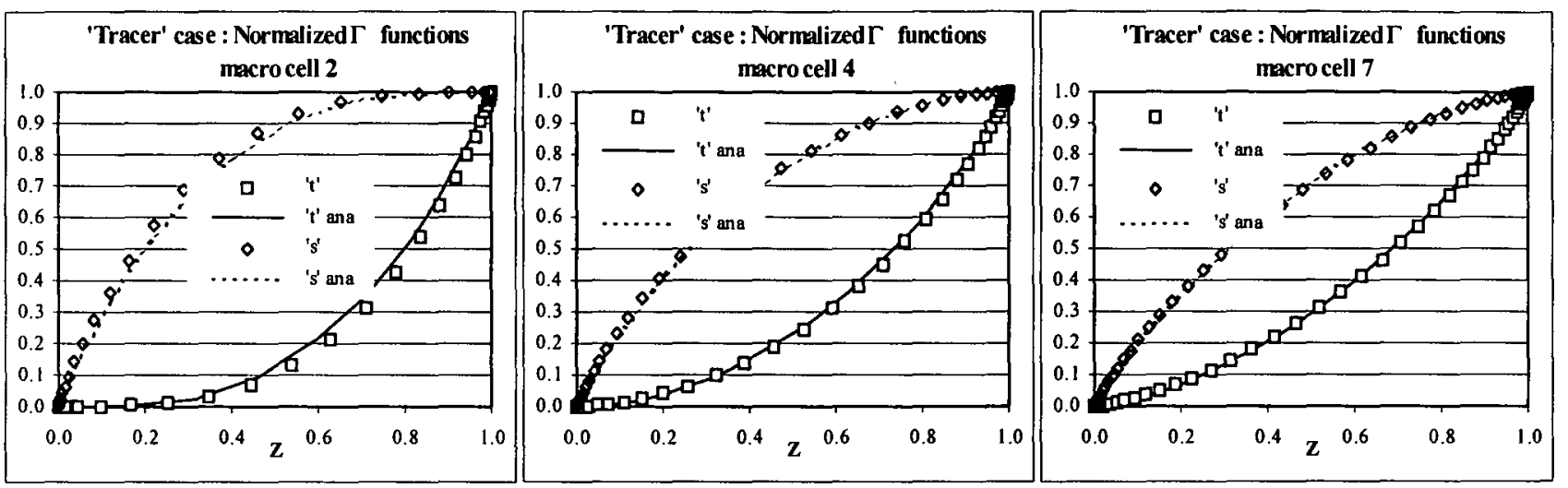

Fig. 7 - 1-D 'Tracer' case analytical simulation Fig. 8 - 1-D 'Tracer' case numerical simulations

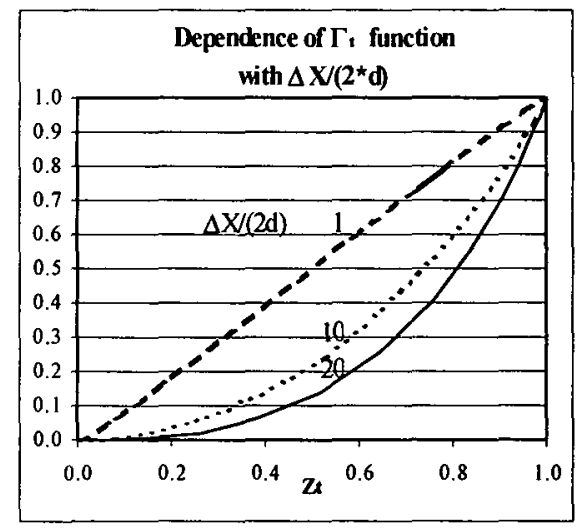

Figure 9

'Tracer' case :

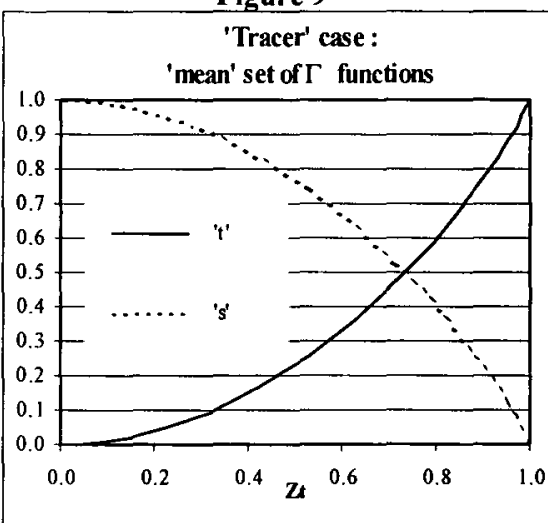

Figure 10

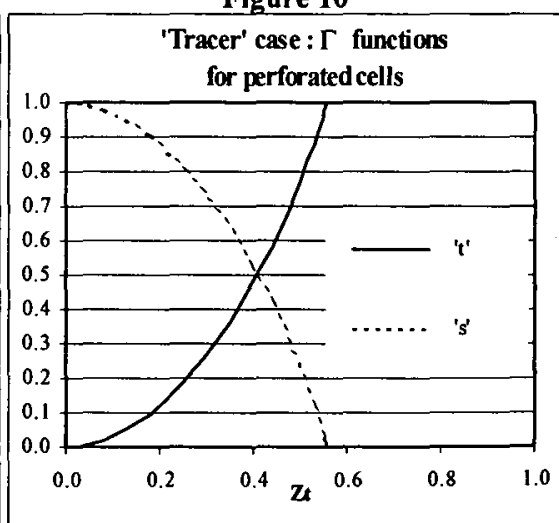

Producer Concentration Profile

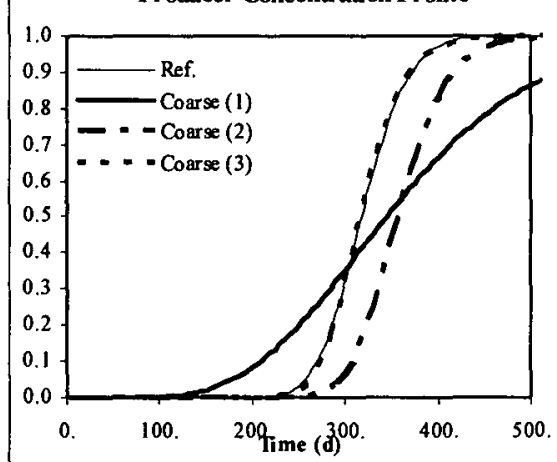

N.B. : hereabove the $\Gamma_{t}$ and $\Gamma_{s}$ functions are plotted vs $Z_{t}$

Figure 11

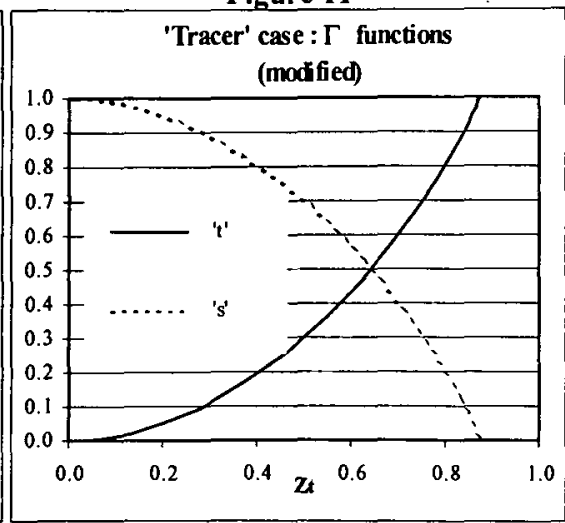


Figure 12 - 1-D 'LG' case : numerical simulations
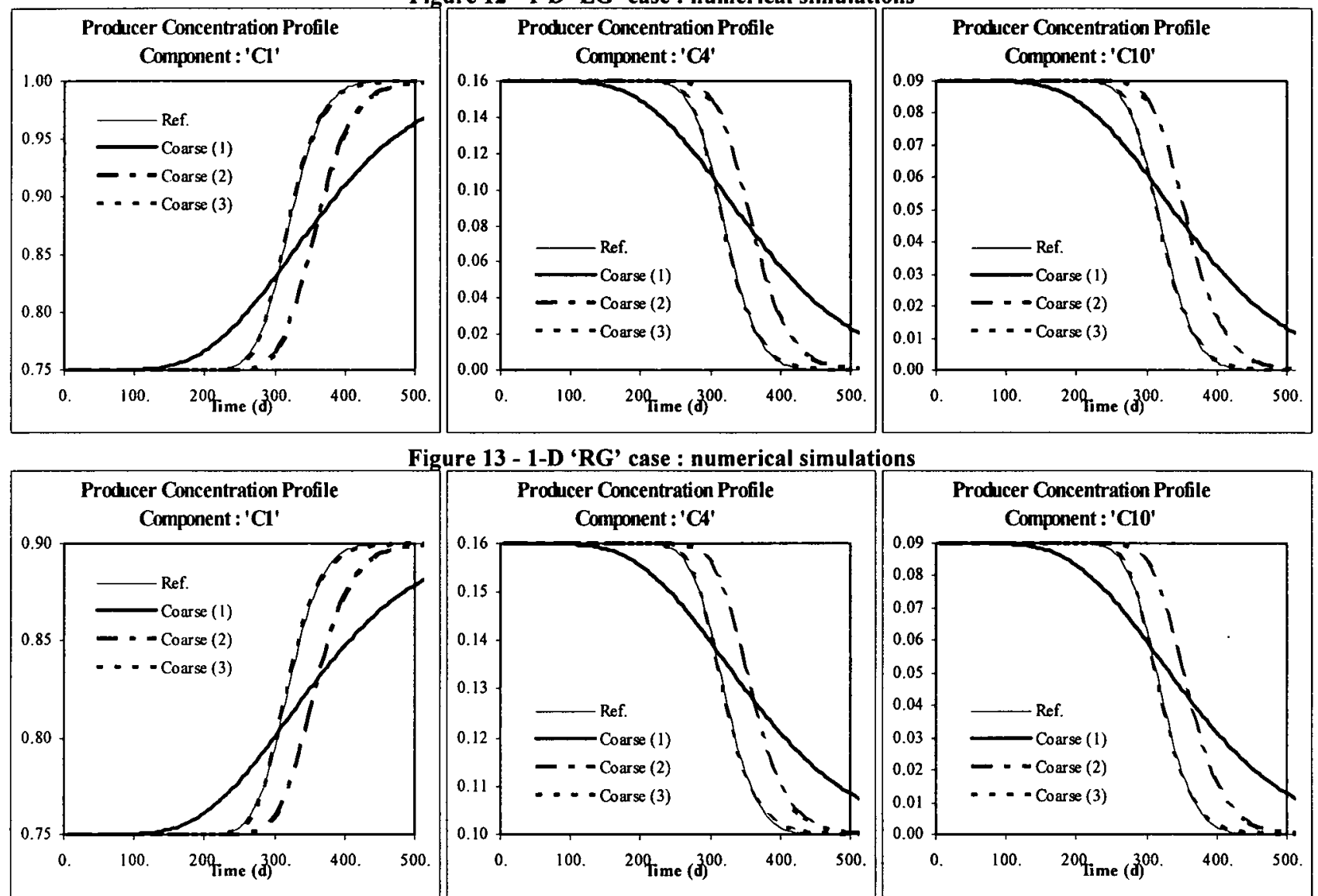

Figure 13 - 1-D 'RG' case : numerical simulations
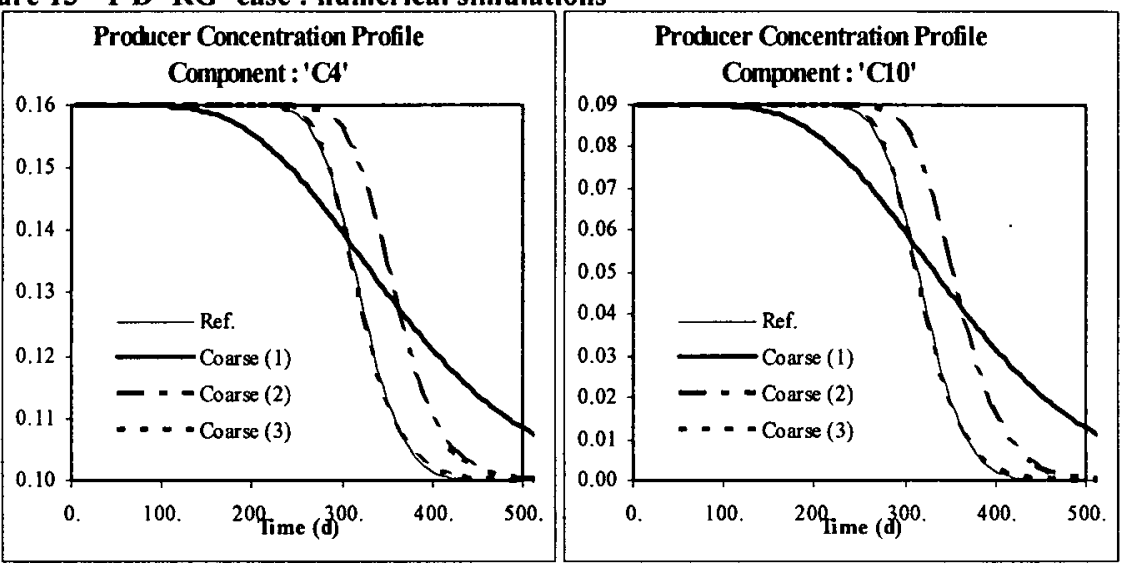

Figure 14 - 2-D 'Tracer' case - Validation run (90 sets of $\Gamma$ functions)

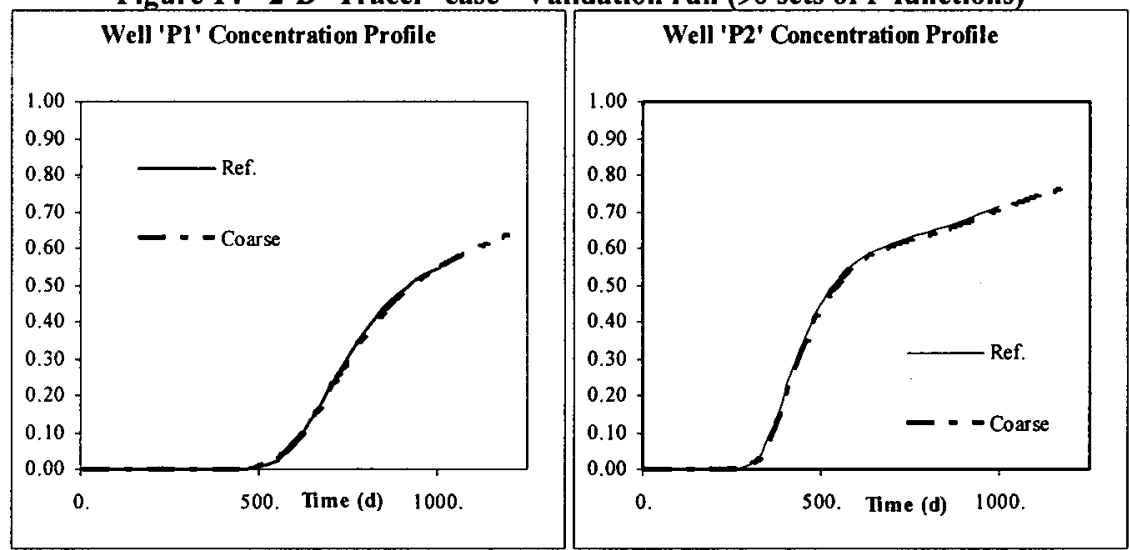

Figure 15 - 2-D permeability map

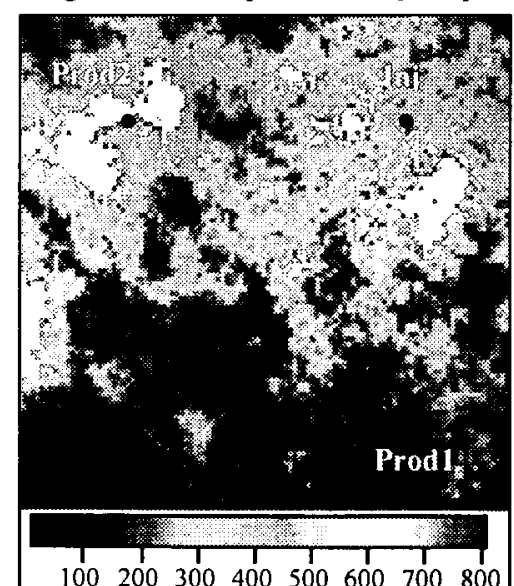

Figure 16 - 2-D 'Tracer' case - Coarse Grid Simulations

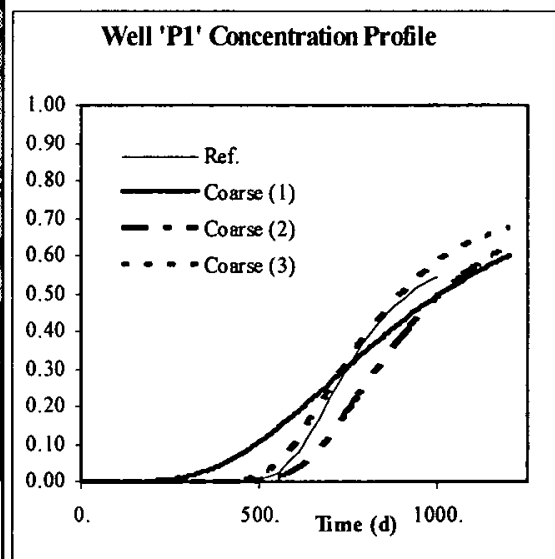


Figure 17 - 2-D 'LG' case : numerical simulations
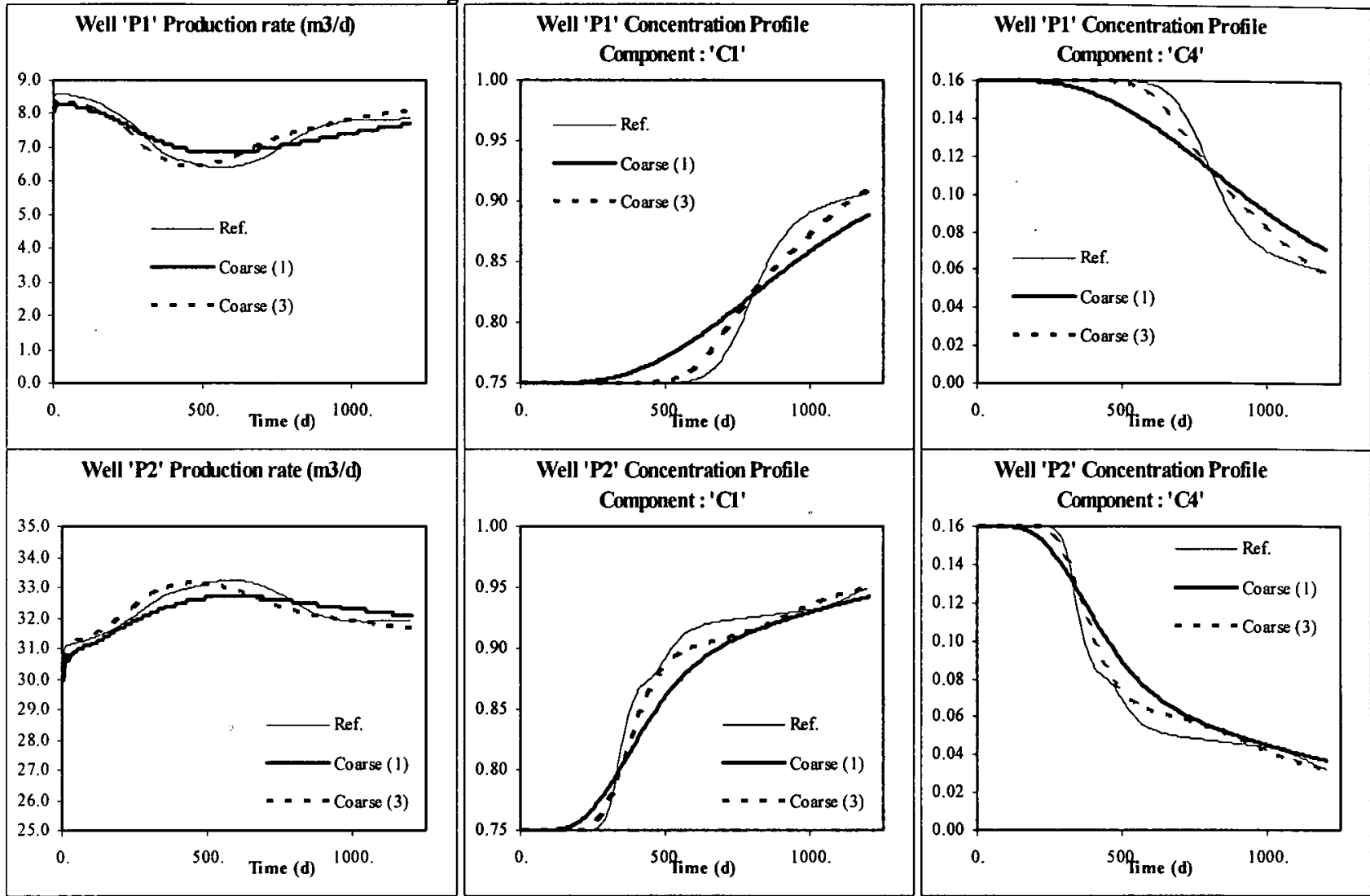

Figure 18 : 1-D ' $O$ ' Case : Fractional Flux Functions
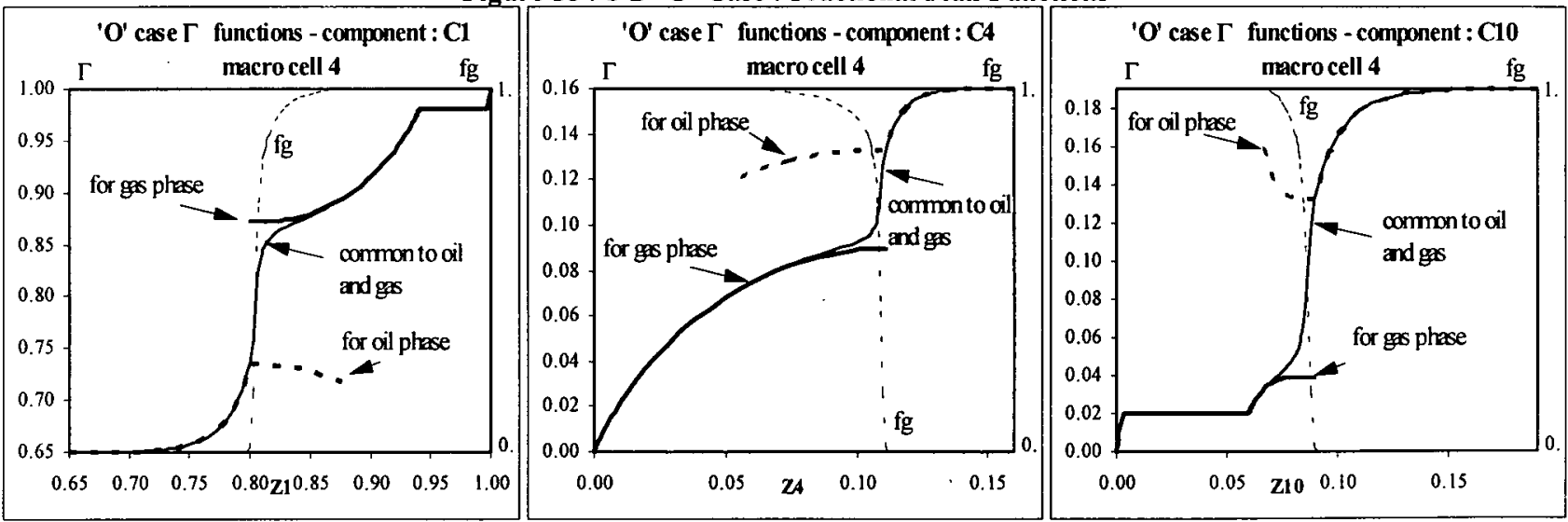

Figure 19

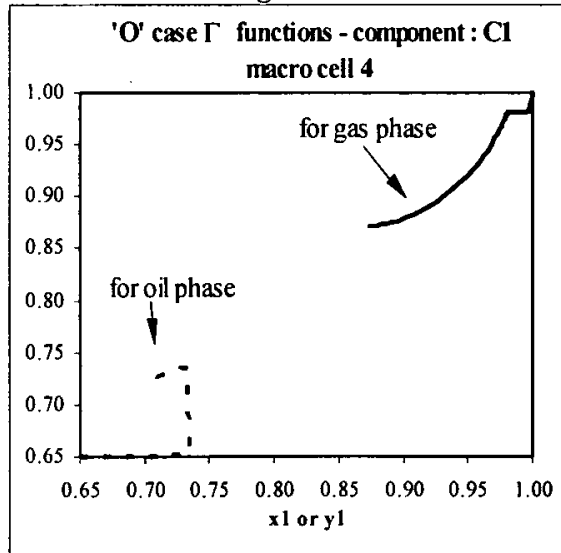

Figure 20 : 1-D ' $O$ ' case : numerical simulations

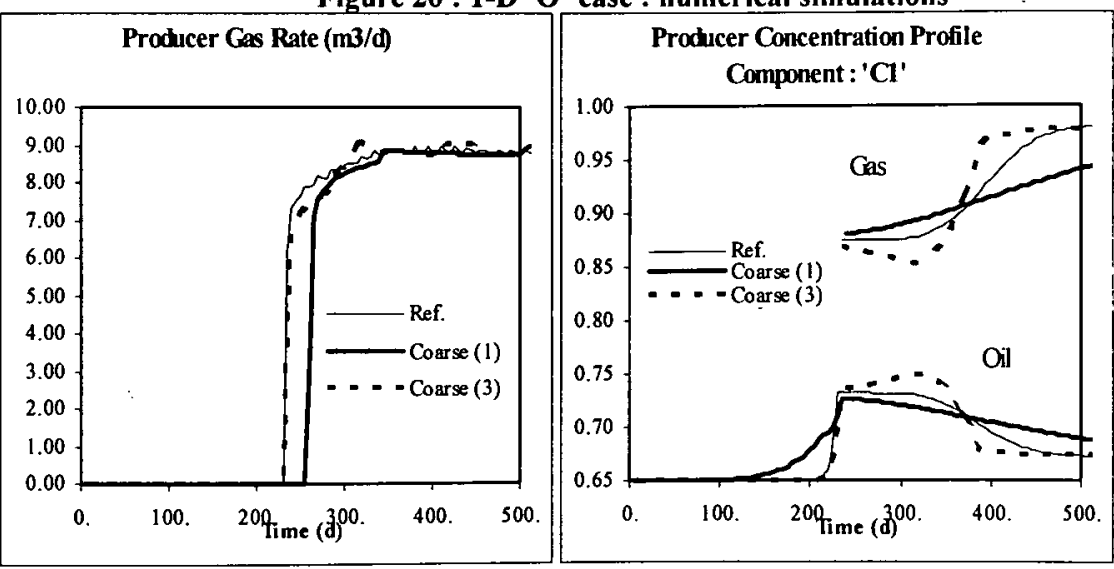


